\title{
Investigation of Channel Interactions in a Nested Hall Thruster Part I: Acceleration Region Velocimetry
}

\author{
Marcel P. Georgin*, Vira Dahliwal†, and Alec D. Gallimore ${ }^{\ddagger}$ \\ University of Michigan, Ann Arbor, MI, 48109, USA
}

\begin{abstract}
Velocity distribution function maps were acquired using laser-induced fluorescence on the centerline of each channel in a two-channel nested Hall thruster. The inner channel velocity distribution maps showed that the acceleration mechanism behaves differently in single and dual channel modes By matching the local thruster pressure through gas injection, the behavior of the dual channel acceleration mechanism can be reproduced in single channel mode. The outer channel velocity distribution maps did not show distinct changes with pressure variation. A difference in the mean exit velocity between dual and single channel modes was measured. This difference in exit velocity could result in increased thrust in dual channel mode.
\end{abstract}

\section{Nomenclature}

$\langle v\rangle \quad$ Mean velocity

E Electric field

v Particle velocity

e Elementary charge

$f(v) \quad$ Velocity distribution function

$I_{D C} \quad$ DC discharge current

$I_{R M S} \quad$ RMS discharge current oscillations

$m_{X e} \quad$ Mass of xenon

$P_{\text {local }}$ Local thruster pressure

$P_{\text {wall }} \quad$ Facility background pressure measured at the wall

$v \quad$ Velocity

*Ph.D. Candidate, Applied Physics, AIAA Student Member.

${ }^{\dagger}$ Research Assistant, Aerospace Engineering

$\ddagger$ Robert J. Vlasic Dean of Engineering, the Richard F. and Eleanor A. Towner Professor of Engineering, and an Arthur F. Thurnau Professor of Aerospace Engineering, and AIAA Fellow. 


\section{Introduction}

$\mathrm{T}$ HE nested Hall thruster (NHT) is an electric propulsion device that builds on traditional Hall thruster designs by concentrically nesting discharge channels. This nested configuration increases the discharge area of the thruster and pushes Hall thruster technology towards higher power. High power Hall thrusters are an enabling technology for new mission architectures such as multi-destination robotic missions and manned missions to Mars. ${ }^{1-3}$. NHTs have several advantages over clusters of Hall thrusters, including an improved power-to-mass ratio as well as a larger throttling range. ${ }^{4,5}$ This technology has advanced dramatically in recent years yielding thrusters that can operate at over $100-\mathrm{kW} .{ }^{6,7}$ Although NHTs have matured in terms of design, few measurements of plasma properties in the channel of an NHT have been made. ${ }^{4}$ To improve the understanding of multi-channel discharges, a laser-induced fluorescence (LIF) velocimetry experiment was conducted on the X2 NHT to characterize the acceleration region.

The X2 is a two channel NHT with a nominal operating power of $5 \mathrm{~kW}$ at $150 \mathrm{~V}$ and is shown in Fig. 1. This thruster was developed by the University of Michigan and the Air Force Research Laboratory as a proof of concept for the nested channel design. This thruster uses a single centrally mounted cathode as the electron source as well as shared magnetic poles. This NHT was designed to operate in either dual channel mode or single channel mode allowing the thruster to throttle between 0.5 and $10 \mathrm{~kW}$. The thruster was initially characterized with thrust measurements, a plume characterization using $E \times B$, Faraday, and Langmuir probes as well as interal measurements with a floating probe. ${ }^{4}$ These previous thrust measurements indicated that the sum of the thrust from each individual channel was less than the thrust measured in dual channel mode at a fixed background pressure. ${ }^{4,8}$ Far-field probe measurements showed that the acceleration voltage and number of multiply charged ions both increase in dual channel mode. Near-field Faraday probe measurements showed that current utilization efficiency also improved in dual channel mode.

Building on this initial body of work on NHTs, LIF was used to measure the velocity distribution function in the acceleration region

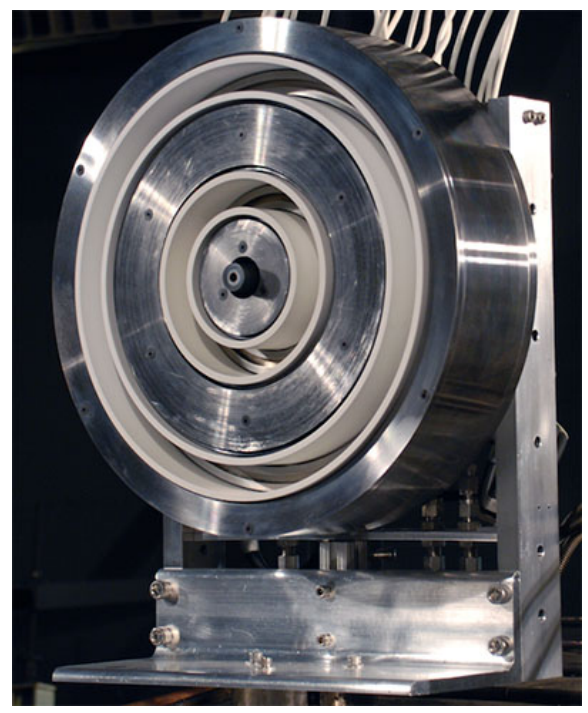

Figure 1: X2 NHT at PEPL of the thruster. Laser-induced fluorescence has been used to measure ion velocity distribution in both the far-field plume and the acceleration regions of Hall thrusters. ${ }^{9,10}$ Using this LIF technique, the velocity distribution of $\mathrm{Xe}^{+}$can be measured as a function of position. These data can then be analyzed to find the shape and position of the accelerating electric field. ${ }^{11}$

Because experiments are operated in a vacuum facility, the background pressure changes depending on the operating condition. For a nested Hall thruster, the facility pressure will be higher in dual channel mode than in single channel mode. It has been shown on the SPT-100 and H6 that background pressure can affect both the performance and the oscillatory mode of a Hall thruster. ${ }^{12,13}$ Additionally, it has been shown on the BHT-600 Hall thruster that changes in the chamber operating pressure can result in a modification of the shape and position of acceleration region. ${ }^{14}$ Since facility background pressure can affect both the acceleration mechanism as well as plasma oscillations, pressure in the facility must be controlled for direct comparison between dual and single channel operation. This work characterizes differences in the acceleration region between single channel and dual channel operation in the X2 NHT using LIF while controlling the chamber pressure.

\section{Experimental Methods}

\section{A. Laser-Induced Fluorescence}

In this work, the traditional LIF scheme for $\mathrm{Xe}^{+}, 5 \mathrm{~d}[4]_{7 / 2} \rightarrow 6 \mathrm{p}[3]_{5 / 2}$, was used to characterize the acceleration region of the X2 NHT. This transition has been widely used in the literature because of its relatively small linewidth $(600 \mathrm{MHz})$ in comparison to the width of the distributions expected in the acceleration region. ${ }^{11,14-17}$ 


\section{B. External Optics}

Figure 2 illustrates the air-side optical setup of the LIF experiment. The laser beam was generated by a tunable diode laser with a tapered amplifier. The beam was sampled at several points by instruments which verify the quality of the beam. First, the beam was sampled by a Fabry-Pérot etalon which was used to verify that the laser has operated in a single mode during a scan. Second, the wavemeter measured the wavelength of the laser beam. Next, the beam was sampled and passed to a photodiode that was used to monitor how much power was produced by the laser. Lastly, the beam was sampled and sent to an optogalvanic cell. This device acted as a stationary wavelength reference for the xenon transition.

After the quality of the beam was verified by these diagnostics, the beam was focused by a lens and was modulated by a mechanical chopper at $2 \mathrm{kHz}$. This allowed the signal to later be recovered by a lock-in amplifier. Once modulated by the

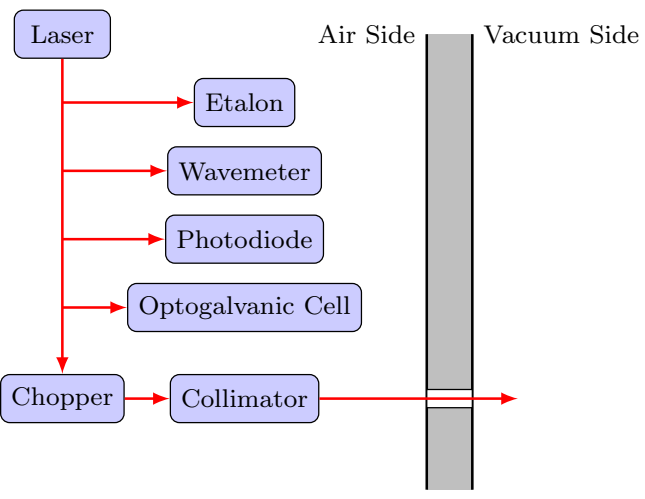

Figure 2: The external setup used for timeaveraged LIF measurements of the X2 acceleration region. chopper, the beam was collimated and passed into the vacuum chamber via fiber optic feedthroughs.

\section{Internal Optics}

Figure 3 depicts the internal setup of the LIF experiment. There were two primary optical elements, the axial laser injection lens and the fluorescence collection lens. Both lenses were coated with anti-reflective coatings to optimize the laser power and maximize the fluorescence signal.

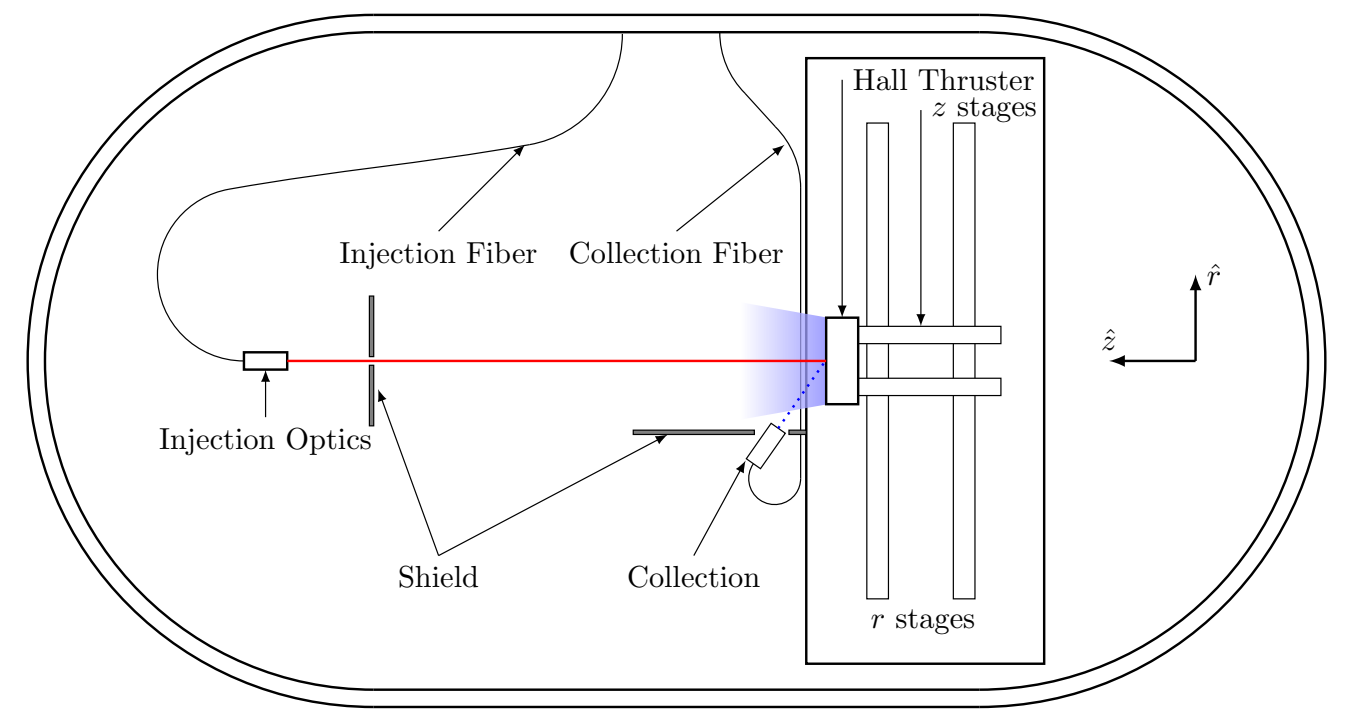

Figure 3: Top-down view of the internal optical setup used for LIF measurements of the X2 acceleration region.

The beam was passed into the vacuum facility by fiber optic and was focused by the injection optic to the interrogation point in the plume of the thruster. The interrogation volume was $1 \mathrm{~mm}^{3}$. A collection lens was mounted at $30^{\circ}$ from the thruster exit plane and was focused on the interrogation volume. At this angle, the collection lens was outside of the thruster plume. The collection lens was used to collect the light fluoresced by the ions when they were excited by the laser. The fluorescence was then sent out of the vacuum chamber by a fiber optic feedthrough. The internal optics were protected by graphite shields. These shields both prevented damage to the lenses from sputtering by the plume and also reduced thermal drift in the position of the optics. For LIF measurements of the inner channel when firing both channels, the setup was slightly modified to keep the collection optics outside the plume of the outer channel. The most notable modification was the change to a longer focal length lens. 
To determine the shape of the acceleration zone, a one dimensional scan needed to be created. To generate this map, the $\mathrm{X} 2$ was placed on $r$ and $z$ motion stages. By moving the thruster in the radial direction, each channel can be interrogated. By moving the thruster in the axial direction a map of the acceleration zone was measured.

\section{Signal Processing}

To measure the signal, the fluorescence is sent out of the vacuum chamber and passed through a monochrometer. This device uses a grating to only permit light at the fluorescence wavelength, $541.9 \pm 1 \mathrm{~nm}$, to pass through to a photomultiplier tube that converts these photons to an electrical signal. This electrical signal was next sent to a lock-in amplifier which was set to lock on the modulation frequency of the mechanical chopper. The signal was recorded and averaged over a time constant, $\tau=300 \mathrm{~ms}$.

\section{E. Operating Conditions \& Test Points}

The X2 was operated using research grade xenon in the Large Vacuum Test Facility (LVTF) at the University of Michigan. LVTF is a cylindrical vacuum chamber which is $9 \mathrm{~m}$ in length and $6 \mathrm{~m}$ in diameter. The thruster was mounted on chamber centerline to minimize the interaction of the plasma with the walls of the facility. A $3 \mathrm{~m} \times 4 \mathrm{~m}$ carbon panel beam dump was placed $3 \mathrm{~m}$ downstream of the thruster to absorb the energy of the ion beam. The facility uses four mechanical roughing pumps and seven cryogenic pumps to achieve a base pressure of $10^{-7}$ Torr.

In this experiment, the thruster was operated at the nominal condition where the inner channel uses 1.5 $\mathrm{kW}$ and the outer channel uses $3.5 \mathrm{~kW}$ for a total power input of $5 \mathrm{~kW}$. The two channels of the X2 can be operated independently or simultaneously without changing magnetic field settings. These features lead to three possible operating conditions. The single channel operating conditions have lower facility background pressure than the dual channel condition. The chamber pressure was controlled by injecting additional xenon gas when making measurements of the single channel operating conditions. Gas was either injected downstream or through the non-operating channel. These injection locations lead to a total of seven test conditions which are shown in Tab. 1. The cathode flow fraction was set to $10 \%$ of the anode flow rate. Because of the change in the cathode flow, the residual gas was flowed through downstream injector to match the background pressure. Chamber pressure was measured at the walls of the facility with an ion gauge. The gauge was located $3 \mathrm{~m}$ radially and $2 \mathrm{~m}$ downstream of the thruster. The local thruster pressure was measured during cold flow testing of the X2. Pressure measurements for all 7 test conditions (note that channel injection cases are the same as dual channel). The local thruster pressure was measured using an internal Stabil ion gauge. The thruster pressure was measured at multiple radial positions by moving the thruster with the motion stages. The inlet of the Stabil ion gauge was located $10 \mathrm{~cm}$ downstream of the thruster exit.

Table 1: Table of test conditions for the X2. Checkmarks indicate location of additional Xe gas injection.

\begin{tabular}{|c||l|c|c|c||c|c|}
\hline Condition & $\begin{array}{l}\text { Operating } \\
\text { Channel }\end{array}$ & Downstream & Outer & Inner & $P_{\text {wall }}[$ Torr] & $P_{\text {local }}$ [Torr] \\
\hline 1$)$ & Dual Channel & & & & $5.5 \times 10^{-5}$ & $1.2 \times 10^{-4}$ \\
\hline 2$)$ & Inner & & & & $1.6 \times 10^{-5}$ & $5.8 \times 10^{-5}$ \\
\hline 3$)$ & Inner & $\checkmark$ & & & $5.5 \times 10^{-5}$ & $7.2 \times 10^{-5}$ \\
\hline 4$)$ & Inner & & $\checkmark$ & & $5.5 \times 10^{-5}$ & $1.2 \times 10^{-4}$ \\
\hline 5$)$ & Outer & $\checkmark$ & & & $3.4 \times 10^{-5}$ & $7.2 \times 10^{-5}$ \\
\hline 6$)$ & Outer & & & $\checkmark$ & $5.5 \times 10^{-5}$ & $7.6 \times 10^{-5}$ \\
\hline 7$)$ & Outer & & & $5.5 \times 10^{-5}$ & $1.2 \times 10^{-4}$ \\
\hline
\end{tabular}

Figure 4 illustrates the operating conditions and depicts the locations at which LIF data were acquired. The location of gas injection for a test condition in Fig. 4 is identified by an arrow and a label. Ion and neutral velocity distribution measurements were acquired at these seven operating conditions. LIF data was collected on the centerline of each channel. Measurements were acquired between $z=-9 \mathrm{~mm}$ and $z=17$ $\mathrm{mm}$, where $z=0 \mathrm{~mm}$ was the exit plane of the thruster. 


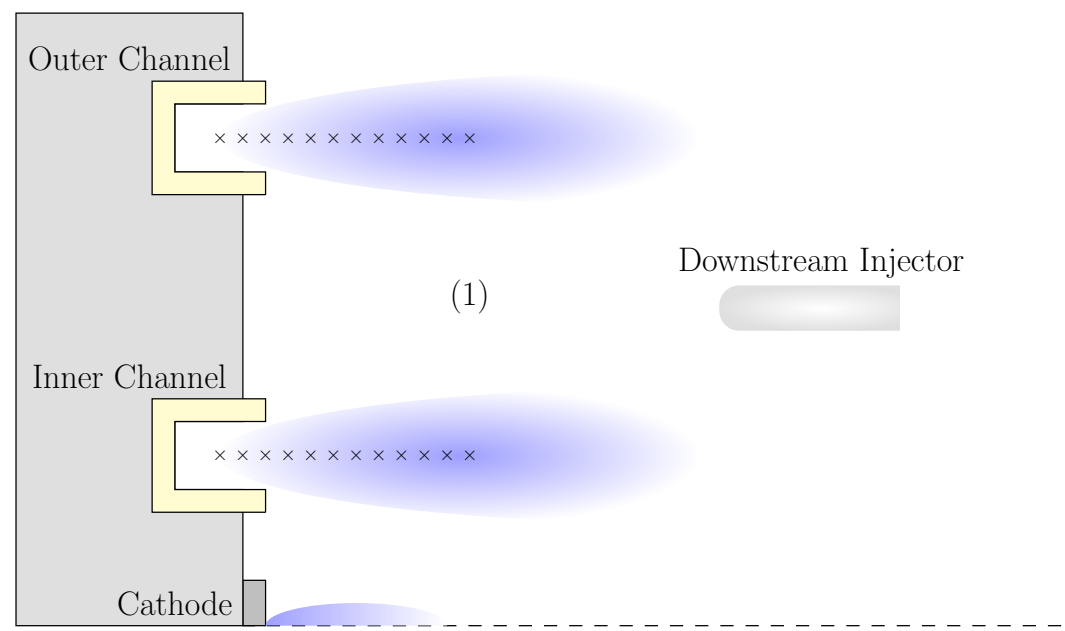

(a) Illustration of LIF measurements for the dual channel operating condition.

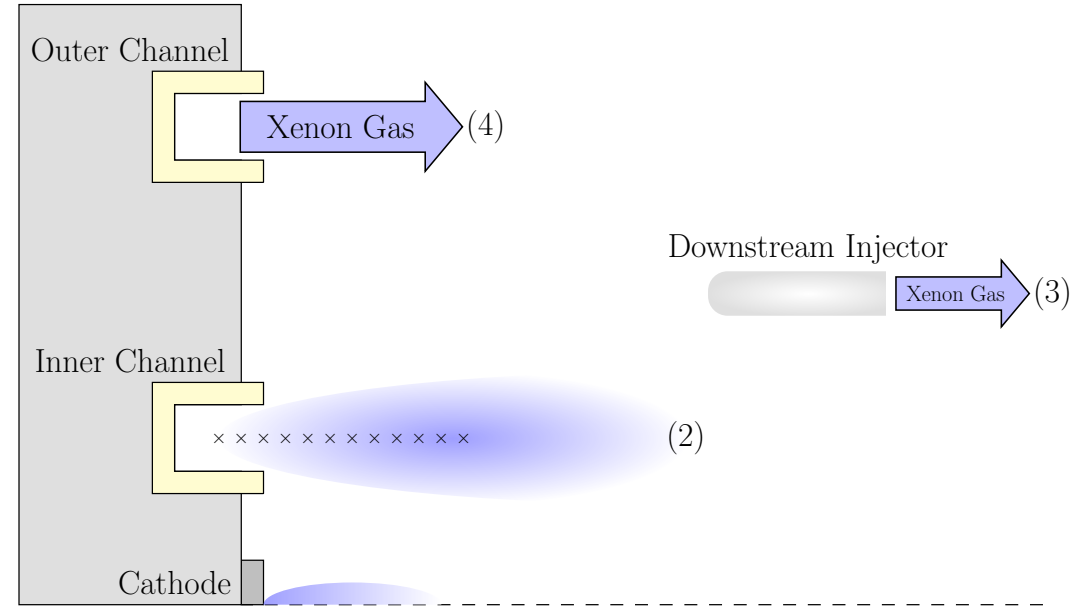

(b) Illustration of LIF measurements for the inner channel operating conditions.

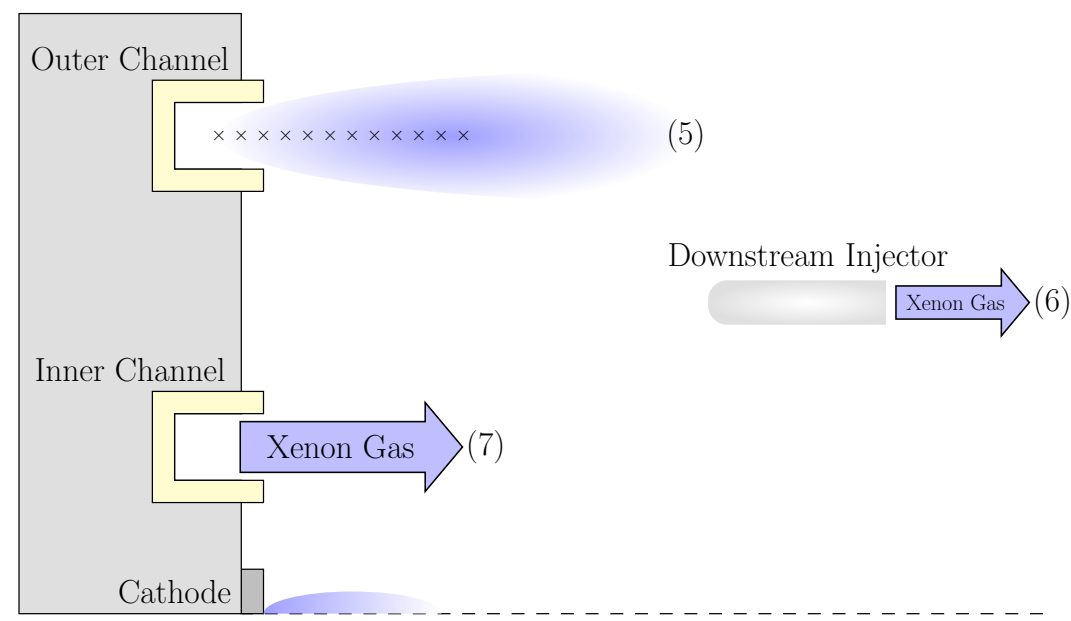

(c) Illustration of LIF measurements for the outer channel operating conditions.

Figure 4: Axisymmetric illustration of the operating conditions and LIF interrogation points. The dashed line is an azimuthal axis of symmetry. 


\section{Results \& Discussion}

\section{A. Local Pressure \& Discharge Oscillations}

Although the chamber pressure was held constant, the local pressure changed between each operating condition. The local pressure was averaged over the face of the thruster and is shown in Tab. 1. Since changes in pressure have been associated with changes in the RMS amplitude discharge current oscillations ${ }^{12}$, the oscillations were measured with a high-speed oscilloscope and Pearson coils. These measurements are plotted as a function of local pressure in Fig. 5. The DC discharge current for the inner and outer channels were 8.9 A and 21.0 A, respectively. In Fig. 5, the RMS discharge current oscillations of the inner channel are an increasing function of the local pressure. The amplitude of the discharge oscillations increases from $1 \%$ to $8 \%$ of the DC discharge. For the outer channel, a clear dependence on pressure is not found because of the difference in the two high pressure conditions (120 $\mu$ Torr). The difference in these data points could be because true thermal steady state may not have been achieved for the outer channel. The range of the discharge current amplitudes for the outer channel is from $4.7 \%$ to $5.7 \%$ of the DC discharge current. Overall, the discharge oscillations on the outer channel were relatively insensitive to pressure, in comparison to the inner channel.

This result ultimately provides further evidence that facility background pressure during Hall thruster testing can impact the amplitude of discharge oscillations. In particular measurements cannot be directly compared to the dual channel mode because of the change in discharge oscillations due to local pressure effects.

\section{B. Ion Velocity Distributions}

The ion velocity distribution was measured to identify the acceleration region in the X2 NHT at various local thruster pressures.

\section{Inner Channel}

Laser-induced fluorescence measurements of the velocity distribution function were acquired at $1 \mathrm{~mm}$ intervals on the inner channel of the X2, however, Fig. 6 only shows a subset for clarity. The velocity distributions are plotted as a function axial position along channel centerline. The dashed line is the peak of the ion beam resolved by a peak-finding algorithm. This algorithm smooths the data and returns the local maximum that is at the highest

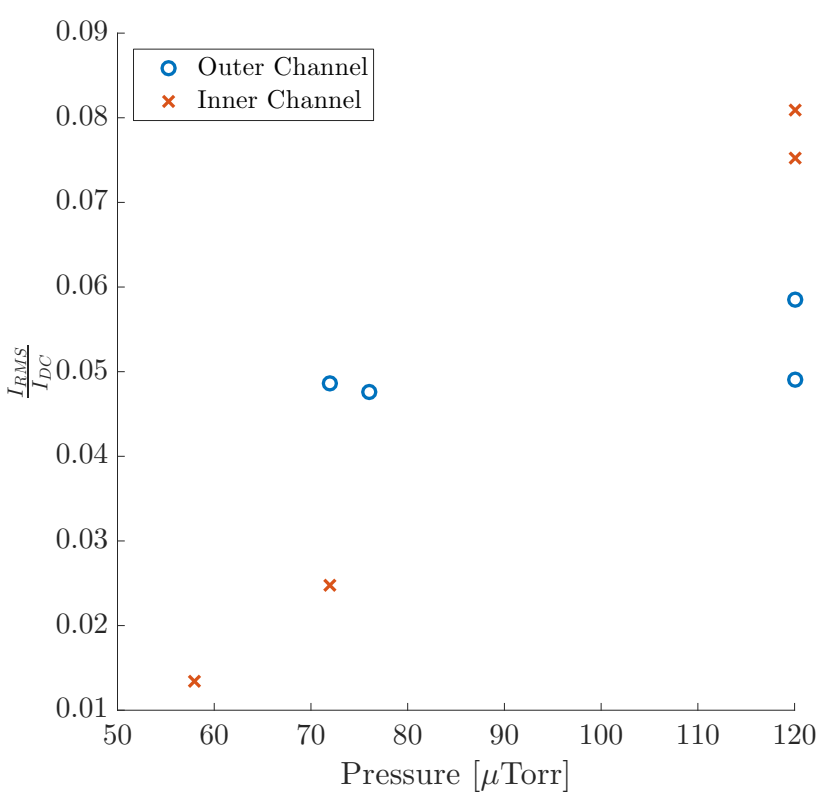

Figure 5: RMS discharge oscillations as a function of local thruster pressure. The uncertainty in the measurement is the size of the data point. velocity. The acceleration region is characterized by the displacement of the ion beam acceleration curve.

Figure $6 \mathrm{a}$ has the lowest local pressure at $5.8 \times 10^{-5}$ Torr. The velocity distribution function in the channel is initially centered around $0 \mathrm{~km} / \mathrm{s}$. The distribution then begins to broaden as the exit plane is approached. Towards the center of the acceleration region, a broad yet distinct ion beam peak separates itself from the slow ion population. Downstream of the primary part of the acceleration region the ion beam dominates the velocity distribution. The velocity curve indicates that the center of the acceleration region is around $5 \mathrm{~mm}$ downstream of the thruster exit plane.

Moving to a higher local pressure by injecting gas downstream of the thruster, many of the features observed in Fig. 6a are also found in Fig. 6b. The key distinction between these conditions is a clear displacement of the acceleration region. The velocity curve in Fig. $6 \mathrm{~b}$ indicates that the center of the acceleration region is around $1 \mathrm{~mm}$ downstream of the exit plane.

Further increasing the local pressure by injecting gas though the outer channel, the velocity distribution functions at the beginning and in the middle of the acceleration region in Fig. 6c have a high velocity ion tail which is not present in Figs. 6a and 6b. In Fig. 6c, rather than observing a distinct ion beam population separate from the slow ion population, the high velocity tail of distribution grows. This process continues 


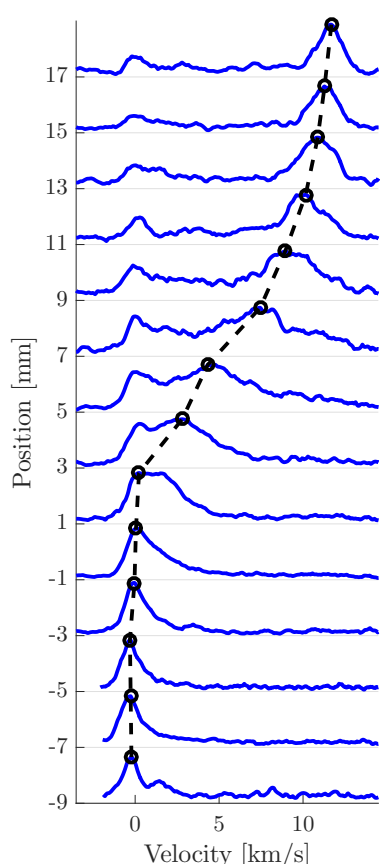

(a) Condition 2 .

Nominal.

$P_{\text {local }}=5.8 \times 10^{-5}$ Torr.

$P_{\text {wall }}=1.6 \times 10^{-5}$ Torr.

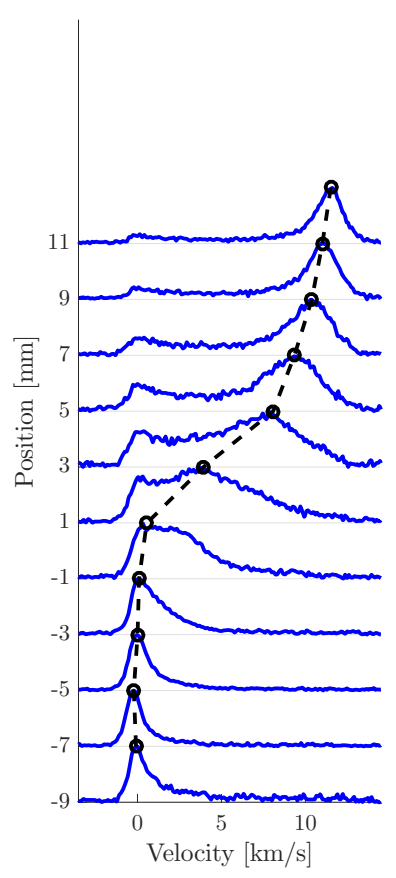

(b) Condition 3.

Downstream Injection.

$P_{\text {local }}=7.2 \times 10^{-5}$ Torr

$P_{\text {wall }}=5.5 \times 10^{-5}$ Torr.

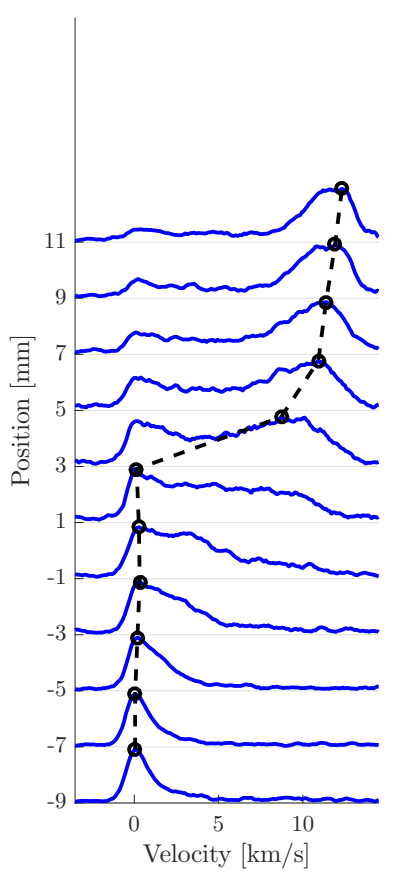

(c) Condition 4.

Outer Channel Injection.

$P_{\text {local }}=1.2 \times 10^{-4}$ Torr.

$P_{\text {wall }}=5.5 \times 10^{-5}$ Torr.

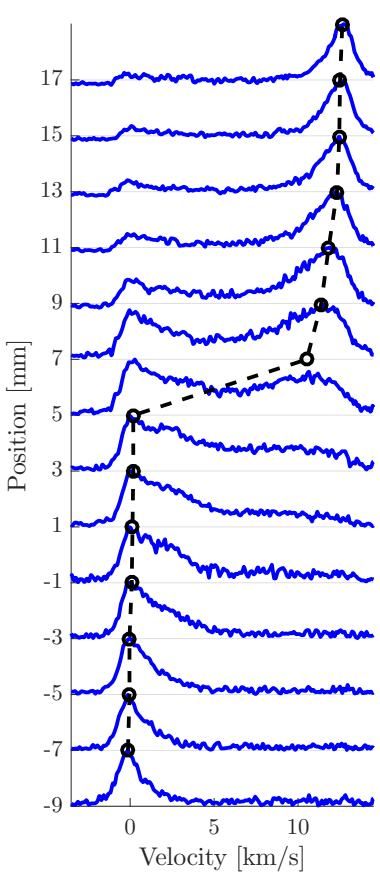

(d) Condition 1.

Dual Channel.

$P_{\text {local }}=1.2 \times 10^{-4}$ Torr.

$P_{\text {wall }}=5.5 \times 10^{-5}$ Torr.

Figure 6: Velocity distribution measurements of the inner channel.

until the a clear ion beam population can eventually be observed. Here, the peak finding algorithm does not properly represent the shape of the acceleration region as a sharp discontinuity exists in the velocity curve. Because no concentrated ion beam population can be found in Fig. 6c, the center of the acceleration region is difficult to determine, however most of the stretching of the velocity distribution function occurs between the -1 and $1 \mathrm{~mm}$, implying that the center of the acceleration region is probably within this area.

Lastly, Fig. 6d shows the evolution of the velocity distribution function along the acceleration region of the inner channel in dual-channel mode. Here, the features are most similar to Fig. 6c. A key difference is that the stretching observed in Fig. 6c now looks more like a slowly rising high-speed ion population. Eventually this population appears as a distinct ion beam population and is detected by the peak-finding algorithm.

There are some features in the velocity distribution functions in Fig. 6that are present in all of the operating conditions. Figures $6 \mathrm{a}-6 \mathrm{~d}$ all have low velocity ion populations which persist out to at least 11 $\mathrm{mm}$ downstream of the exit plane. Furthermore, the distribution near the center of the acceleration region can be extremely bi-modal, exhibiting both a significant population of slow and beam ions.

Combining the results from Figs. 5 and 6 , a picture of how the local thruster pressure is affecting the acceleration region of the inner channel. As local thruster pressure increases on the inner channel, the amplitude of the discharge oscillations increases. These oscillations can be correlated to the different ways that the ion beam is forming between the test conditions. When the discharge oscillations are relatively small (test conditions 2 and 3), the ion beam population separates from the slow ions and is easily identified. However, when discharge oscillations have increased (conditions 4 and 1), the acceleration of the ion beam population can be described by a slow rise in high-speed ions. While a direct cause and effect measurement was not made between a change in discharge oscillations and a change in observed acceleration mechanism, this strong correlation warrants further investigation. Time-resolved LIF techniques can be used to further examine the effects of discharge oscillations on the acceleration mechanism. 


\section{Outer Channel}

Like the inner channel, velocity distribution data was acquired a $1 \mathrm{~mm}$ intervals and Fig. 7 shows a subset of the VDF measurements for clarity. The distributions are plotted as a function of axial position along the outer channel centerline. The dashed line represents the peak of the ion beam.

The ion velocity distribution maps in Fig. 7 all have many similar features. Figures $7 \mathrm{~b}-7 \mathrm{~d}$ all exhibit slow ion populations which persist from $-9 \mathrm{~mm}$ in the channel to beyond the center of the acceleration region. Downstream of the center, the slow ion population is small compared to the number of ions in the beam. All of these velocity distribution maps have an acceleration mechanism which can be described as the formation of an ion beam which separates itself from the slow ion population. The ion beam is very broad in the center of the acceleration region, but the distribution tightens at the exit of the acceleration region.

Although there are many similarities between the test conditions, there are a few important differences. First, the slow ion peak in the nominal and downstream injection conditions, (Figs. 7a and 7b) is consistently smaller than the slow ion peak in the channel injection and dual channel conditions (Figs. 7c and 7d). In other words, as the local pressure is increased the slow ion population becomes more prominent. Second, there is a small upstream shift in the acceleration region for conditions 1 and 7 (high local pressure) compared to conditions 5 and 6 (lower local pressure).

The potential impact of discharge current oscillations on the acceleration mechanism are less prominent on the outer channel, in comparison with the inner channel. This may simply be due to the limited pumping speed of the facility not permitting lower operating pressures and presumably smaller amplitude discharge current oscillations.

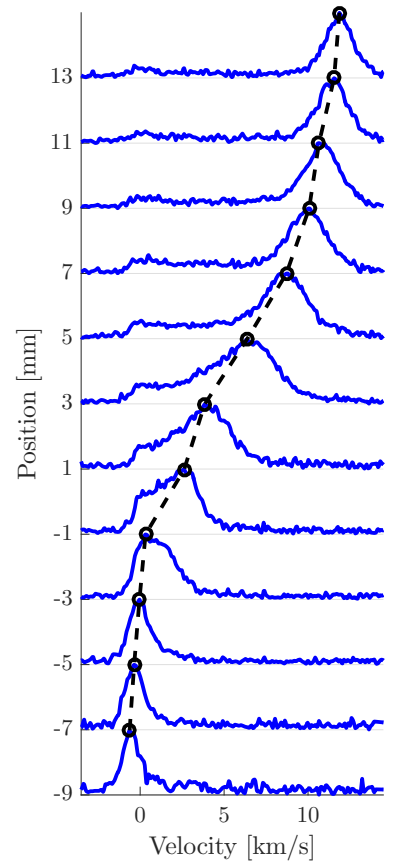

(a) Condition 5 .

Nominal.

$P_{\text {local }}=7.2 \times 10^{-5}$ Torr.

$P_{\text {wall }}=3.4 \times 10^{-5}$ Torr.

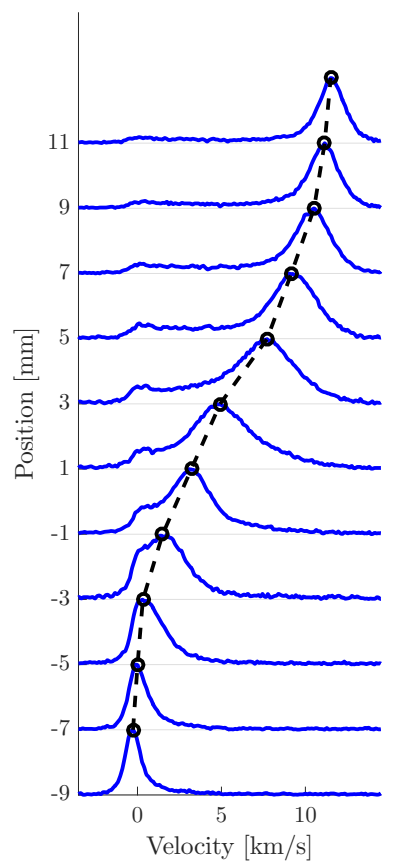

(b) Condition 6 .

Downstream Injection.

$P_{\text {local }}=7.6 \times 10^{-5}$ Torr.

$P_{\text {wall }}=5.5 \times 10^{-5}$ Torr.

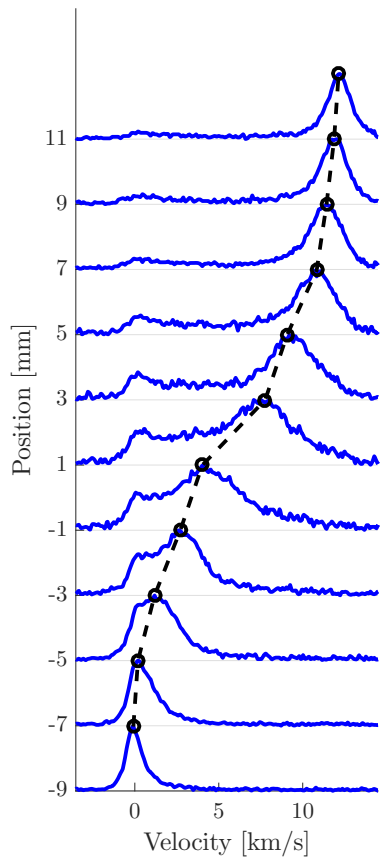

(c) Condition 7.

Inner Channel Injection.

$P_{\text {local }}=1.2 \times 10^{-4}$ Torr.

$P_{\text {wall }}=5.5 \times 10^{-5}$ Torr.

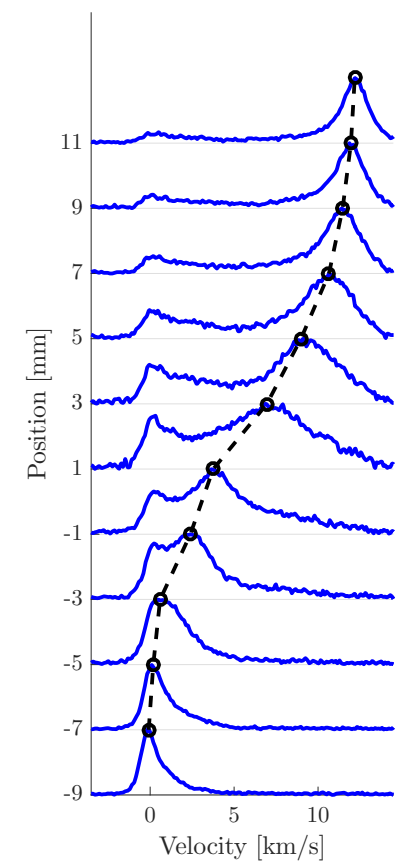

(d) Condition 1.

Dual Channel.

$P_{\text {local }}=1.2 \times 10^{-4}$ Torr.

$P_{\text {wall }}=5.5 \times 10^{-5}$ Torr.

Figure 7: Velocity distribution measurements of the outer channel of the X2 NHT.

\section{Impact On Thruster Performance}

Thus far, the acceleration region has been described by velocity distribution maps and by a velocity curve that tracks the peak of the ion beam population. One weakness of this method is the dependence on a local maximum to find the ion beam velocity. This definition of a velocity curve, however, is useful when trying 
to determine the electric field in the channel through:

$$
\mathbf{E}=-\frac{e}{m_{X e}} \mathbf{v} \cdot \nabla \mathbf{v}
$$

where $\mathbf{E}$ is the electric field, $e$ is the elementary charge, $m_{X e}$ is the mass of xenon and $\mathbf{v}$ is the particle velocity. Another quantity of interest is the mean of the distribution, which can be computed using:

$$
\langle v\rangle=\int_{-\infty}^{\infty} v f(v) d v
$$

where $f(v)$ is the velocity distribution function and $\langle v\rangle$ is the mean velocity. The velocity curve produced using Eqn. (2) is in more robust than the peak-finding algorithm because it does not rely on the presence of local maxima to generate the curve. Furthermore, it is more accurate to use this quantity when considering performance because the thrust is ultimately a result of the mean exit velocity. By examining and comparing these two velocity curves, a better understanding of how the changes in local pressure may be impacting the performance of each channel may be developed. Below, these two types of velocity curves are examined for the different test cases.

\section{Inner Channel}

Figure $8 \mathrm{a}$ presents the peak ion beam velocity as a function of position while Fig. $8 \mathrm{~b}$ is the electric field computed using the results from Fig. 8a and Eqn. (1).

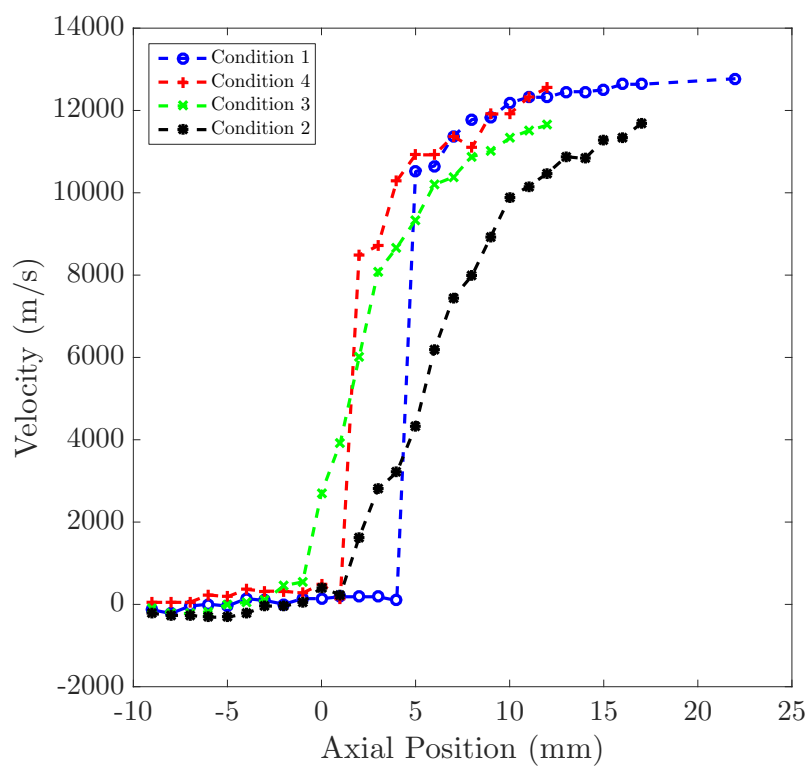

(a) Ion beam velocity curves for the inner channel.

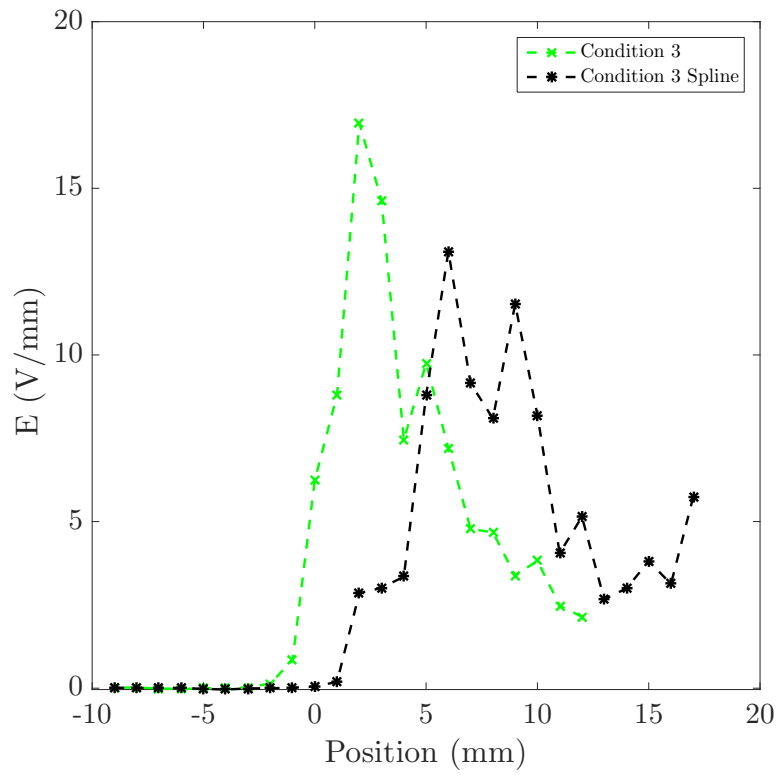

(b) Computed electric field.

Figure 8: Inner channel velocity curve and resulting electric field.

Many of the features that were observed in Fig. 6, such as position and shape, are present in Fig. 8a. As the local pressure is increased, the acceleration region is moved upstream of the exit plane. Similar displacements due to changes in facility pressure have been observed in the literature. ${ }^{14}$ Examining the discontinuity in the dual channel and channel injection test points (conditions 1 and 4), the peak-finding algorithm cannot find the ion beam velocity when there is no local maximum. This causes the ion beam to be misidentified as the slow ion population. This results in the discontinuity observed in conditions 1 and 4 in Fig. 8a. Although the discontinuity largely obscures the acceleration region, the shift in the position can still be clearly observed after the ion beam peak has formed. 
In the context of performance, the ion beam exit velocity gives a sense of how much of the applied voltage is being used to accelerate ions. As the local pressure increases the exit velocity appears to increase, although the limited data set cannot with confirm this in the far field. However, this result is corroborated by plasma potential measurements by Liang (at a different operating power) which showed a difference in voltage utilization between dual and single channel mode. ${ }^{4}$

Figure $8 \mathrm{~b}$ shows the electric field computed directly from the data in Fig. 8a and the field computed using a smooth spline fit of the velocity curves in Fig. 8a. The electric field could only be computed for conditions 3 and 2 because of the discontinuity in conditions 1 and 4 . Figure $8 \mathrm{~b}$ shows that the higher chamber pressure in condition 3 results in a larger electric field and a $4 \mathrm{~mm}$ upstream shift in the peak electric field in comparison to condition 1 . The position and magnitude of the electric field can cause the divergence angle of the thruster to change, possibly leading to changes in performance.

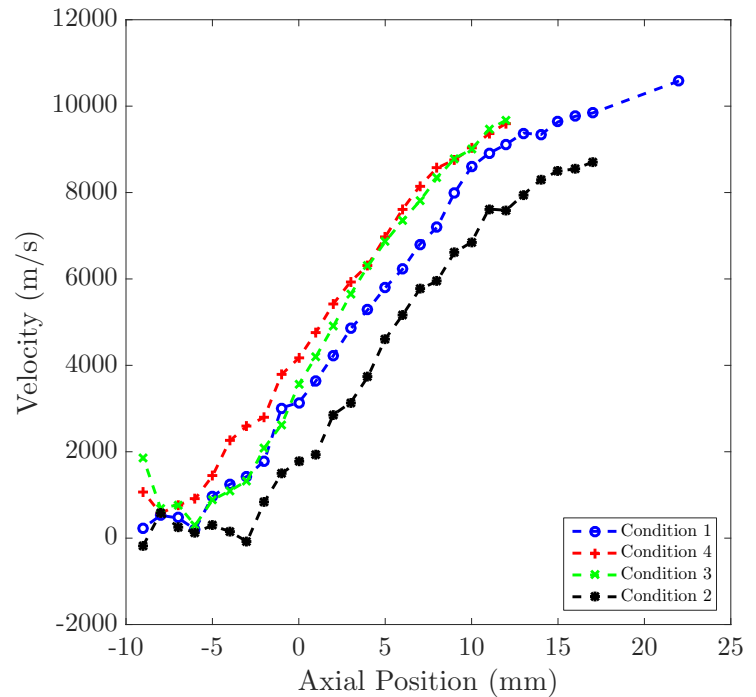

Figure 9: Mean ion velocity curves for the inner channel.

Since the electric field in condition 3 has a larger amplitude and is closer to the channel exit, an improved thrust measurement would be expected because of a reduced divergence angle.

Figure 9 shows the mean of the velocity distribution, computed using Eqn. (2), as a function of position. In the context of performance, the mean exit velocity is what should determine the average exhaust velocity of the thruster and therefore impact the thrust produced. Figure 9 shows that the nominal case for the inner channel (condition 1) has a slower mean exit velocity by about $1000 \mathrm{~m} / \mathrm{s}$, within the scope of this experiment. This result shows that a higher local pressure can cause a higher mean exhaust velocity. Ultimately it is important when testing NHTs to control the chamber background pressure, otherwise the exhaust velocity and therefore the thrust may be affected.

\section{Outer Channel}

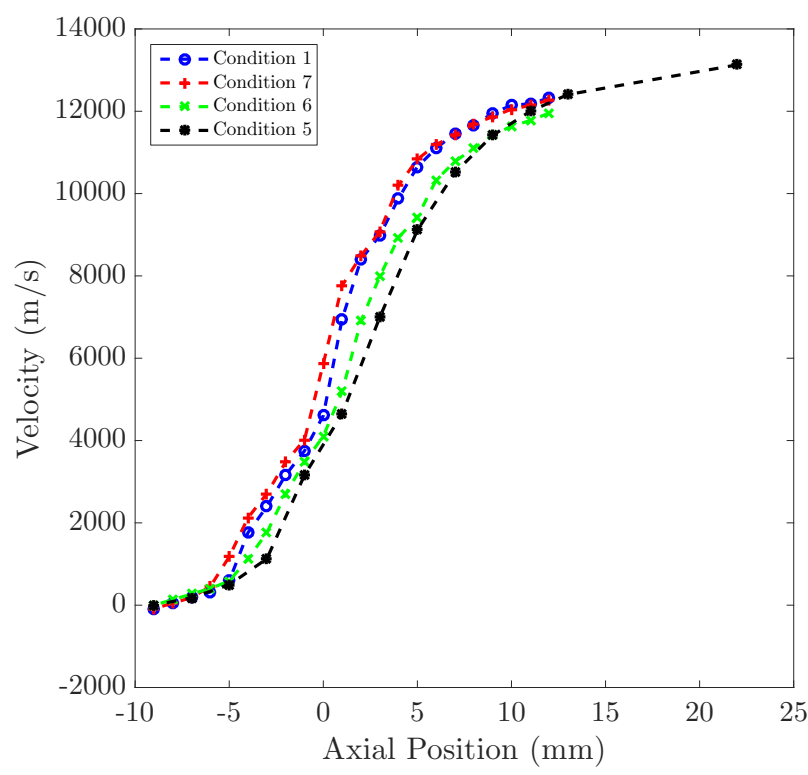

(a) Ion beam velocity curves for outer channel.

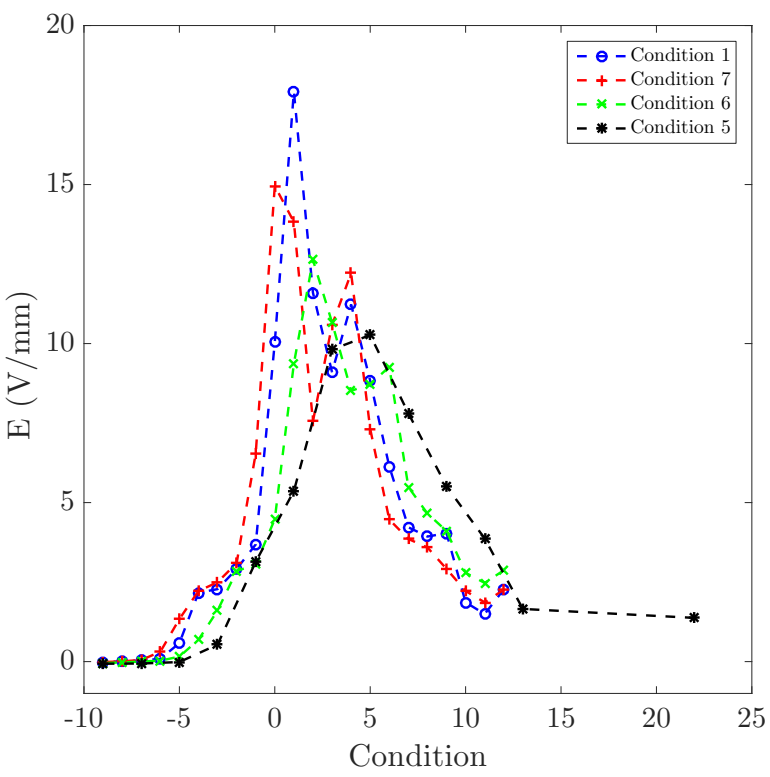

(b) Computed electric field.

Figure 10: Outer channel velocity curves and resulting electric field. 
Conducting a similar analysis for the outer channel, Fig. 10a shows small upstream shifts in the position of the acceleration region as the local pressure is increased between conditions. Most notably, the outer channel cases all have the same exit velocity for the ion beam. From the curves in Fig. 10a, the electric field can be computed using Eqn. (1). The electric field shown in Fig. 10b was computed using both the raw data and a spline fit to the velocity curves in Fig. 10a. In Fig. 10b, the electric field amplitude increases and the distribution tightens as the local pressure is increased. These trends in the electric field profile agree with the observations Fig. 8b. Furthermore, the results on the outer channel indicate that the electric field produced in dual channel mode can be reproduced in single channel mode by correcting for the difference in local thruster pressure.

Lastly, the mean ion velocity as a function of position was computed and is shown in Fig. 11. The nominal condition for the outer channel (condition 5) was at the lowest pressure and the average exit velocity was about

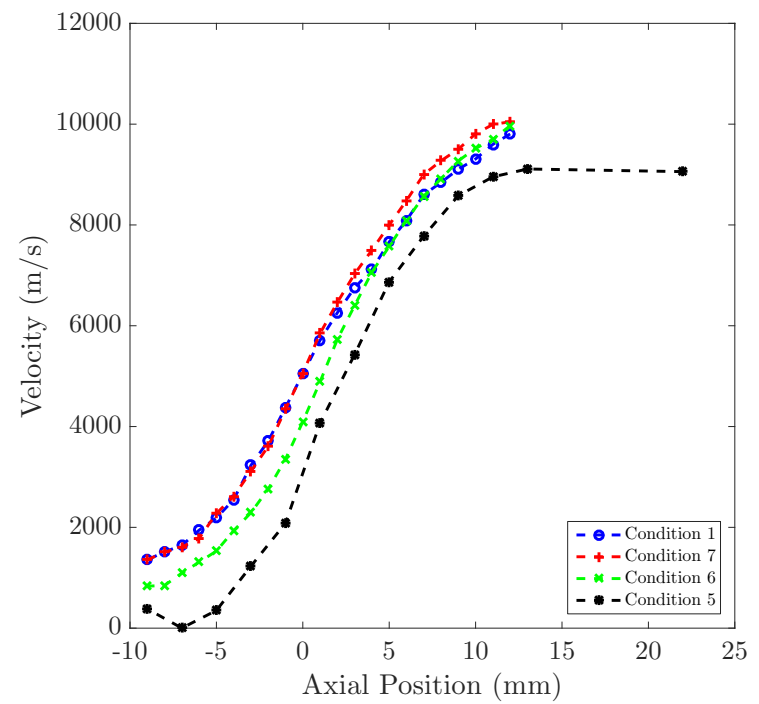

Figure 11: Mean ion velocity curves for outer channel. $900 \mathrm{~m} / \mathrm{s}$ slower than the higher local pressure conditions within the bounds of this experiment. The lower mean exit velocity implies that the thrust produced in single channel mode should be lower than the thrust produced in dual channel mode, for the same mass flow.

\section{Conclusion}

The RMS discharge current oscillations were mapped as a function of local thruster pressure. These measurements showed that increased local thruster pressure leads to larger discharge current oscillations.

Next, velocity distribution function maps were measured on the inner and outer channels. For the inner channel, qualitative differences in the velocity distribution functions were observed. In single channel mode the acceleration potential separates the ion beam from the slow ion population. In dual channel mode, the acceleration mechanism is better described by a rising high speed ion population. By matching local pressure, the dual channel acceleration mechanism is recovered in the single channel mode. For the outer channel, only a small difference in the acceleration region was detected.

Lastly, the velocity distribution functions were analyzed to determine the ion beam and the mean ion velocity curves. This analysis showed that increased local pressure moves the acceleration region upstream of the thruster exit plane. The ion beam velocity curve was used to compute the electric field. The results indicate that by correcting for the local pressure, the electric field produced in dual channel mode can be reproduced in single channel mode by matching the local pressure. The mean exit velocity was found to be lower in single channel mode for both channels. This could result in a difference in the thrust between dual and single channel modes.

\section{Acknowledgments}

This work was funded by NASA Space Technology Research Fellowship grant NNX15AQ37H. The authors would like to thank Timothy B. Smith and Chris Durot for their assistance with setting up the laser-induced fluorescence experiment as well as Sarah E. Cusson for her help with the internal pressure measurements.

\section{References}

1 Manzella, D., Oh, D., and Aadland, R., "Hall Thruster Technology for NASA Science Missions," Tuscon, Arizona, USA, 2005.

2 Jacobson, D. T., John, Jeremy, Kamhawi, Hani, Manzella, David, and Peterson, Peter, "An Overview of Hall Thruster Development at NASA's John H. Glenn Research Center," Tuscon, Arizona, USA, 2005. 
${ }^{3}$ Sankaran, K., Cassady, L., Kodys, A. D., and Choueiri, E. Y., "A survey of propulsion options for cargo and piloted missions to Mars," Annals of the New York Academy of Sciences, Vol. 1017, No. 1, 2004, pp. $450-467$.

${ }^{4}$ Liang, R., The combination of two concentric discharge channels into a nested hall-effect thruster, Ph.D. thesis, The University of Michigan, 2013.

5 Florenz, R., Gallimore, A. D., and Peterson, P. Y., "Developmental status of a 100-kW class laboratory nested channel hall thruster," 32nd International Electric Propulsion Conference, Wiesbaden, Germany, 2011, pp. 1-9.

${ }^{6}$ Florenz, R. E., Hall, S. J., Gallimore, A. D., Kamhawi, H., Griffiths, C. M., Brown, D. L., Hofer, R. R., and Polk, J. E., "First Firing of a 100-kW Nested-channel Hall Thruster," Tech. rep., DTIC Document, 2013.

7 Hall, S., Florenz, R. E., Gallimore, A. D., Kamhawi, H., Brown, D. L., Polk, J. E., Goebel, D., and Hofer, R. R., "Implementation and Initial Validation of a 100-kW Class Nested-channel Hall Thruster," 2014.

8 Cusson, S., Dale, E. T., and Gallimore, A. D., "Investigation of Channel Interactions in a Nested Hall Thruster Part II: Probes and Performance," Salt Lake City, Utah, USA, July 2016.

9 Smith, T. B., Hermanz, D. A., Gallimorex, A. D., and Drake, R. P., "Deconvolution of axial velocity distributions from Hall thruster LIF spectra," Pasadena, California, USA, 2001.

10 Huang, W., Gallimore, A. D., and Smith, T. B., "Interior and Near-Wall Ion Velocity Distribution Functions in the H6 Hall Thruster," Journal of Propulsion and Power, Vol. 29, No. 5, 2013, pp. 11461154 .

11 Mazouffre, S., "Laser-induced fluorescence diagnostics of the cross-field discharge of Hall thrusters," Plasma Sources Science and Technology, Vol. 22, No. 1, 2013, pp. 013001.

12 Randolph, T., Kim, V., Kaufman, H., Kozubsky, K., Zhurin, V. V., and Day, M., "Facility effects on stationary plasma thruster testing," 23rd International Electric Propulsion Conference, 1993, pp. 13-16.

13 Reid, B. M., "Empirically-Derived Corrections for Facility Effects in Performance and Plume Measurements of Hall Thrusters," Kobe, Japan, July 2015.

14 Nakles, M. R. and Hargus, W. A., "Background Pressure Effects on Ion Velocity Distribution Within a Medium-Power Hall Thruster," Journal of Propulsion and Power, Vol. 27, No. 4, 2011, pp. 737-743.

15 W. A. Hargus, J. and Cappelli, M. A., "Laser-induced fluorescence measurements of velocity within a Hall discharge," Applied Physics B, Vol. 72, No. 8, 2001, pp. 961-969.

16 Hargus, W. A. and Cappelli, M. A., "Interior and exterior laser-induced fluorescence and plasma measurements within a Hall thruster," Journal of Propulsion and Power, Vol. 18, No. 1, 2002, pp. 159-168.

17 Hargus, W. A. and Charles, C. S., "Near exit plane velocity field of a 200-Watt Hall thruster," Journal of Propulsion and Power, Vol. 24, No. 1, 2008, pp. 127-133. 